\title{
Remineralizing Efficacy of a CPP-ACP Cream on Enamel Caries Lesions in situ
}

\author{
Hendrik Meyer-Lueckel ${ }^{a}$ Richard J. Wierichs ${ }^{a}$ Timo Schellwien ${ }^{b}$ \\ Sebastian Paris ${ }^{c}$ \\ aDepartment of Operative Dentistry, Periodontology and Preventive Dentistry, RWTH Aachen University, \\ Aachen, ${ }^{b}$ Clinic for Operative Dentistry and Periodontology, School of Dental Medicine, Christian-Albrechts- \\ Universität zu Kiel, Kiel, and ' Department of Operative and Preventive Dentistry, Charité - Universitätsmedizin \\ Berlin, Berlin, Germany
}

\section{Key Words}

Calcium · Casein phosphopeptide · CPP-ACP · Fluoride · GC Tooth Mousse · In situ model $\cdot$ MI Paste $\cdot$ Phosphate $\cdot$ Remineralization

\begin{abstract}
The aim of this double-blind, randomized, cross-over in situ study was to compare the remineralizing effects induced by the application of casein phosphopeptide-stabilized amorphous calcium phosphate complexes (CPP-ACP)-containing cream (without fluoride) after the use of fluoride toothpaste with the prolonged use of fluoride toothpaste on enamel caries lesions in situ. During each of three experimental legs of 4 weeks, 13 participants wore intra-oral mandibular appliances with 8 pre-demineralized bovine enamel specimens in the vestibular flanges mimicking either 'easily cleanable' or 'proximal' surfaces $(n=312)$. The three randomly allocated treatments were as follows: (1) application of CPP-ACP-containing cream (GC Tooth Mouse, non-fluoride) after the use of fluoride toothpaste (1,400 ppm NaF; TM), (2) prolonged application of fluoride toothpaste (1,400 ppm NaF; positive control, PC) and (3) prolonged application of fluoride-free toothpaste (negative control, NC). Additionally, one of each of the two flanges was brushed twice daily with the respective toothpaste. The differences in integrated mineral loss as
\end{abstract}

assessed by transversal microradiography were calculated between values before and after the in situ period. Changes in mineral loss were analysed for those pairs of subgroups differing in only one of the three factors (intervention, brushing and position). The PC treatment induced a significantly higher mineral gain compared with the TM and NC treatments. No significant differences between TM and NC for both positions were observed. In conclusion, the additional use of a CPP-ACP-containing cream seems to be less efficacious in remineralizing caries lesions than the prolonged application of fluoride toothpaste.

() 2014 S. Karger AG, Basel

The formation of caries lesions can be avoided with non-invasive interventions, i.e. oral hygiene education and adequate use of fluorides. Fluorides are also capable of remineralizing mainly the surface area of non-cavitated caries lesions. However, current non-invasive interventions do not seem to be sufficient to completely avoid the formation as well as the progression of caries lesion in the population [Paris et al., 2012].

For this purpose the use of casein phosphopeptide-stabilized amorphous calcium phosphate complexes (CPPACP) has been proposed. Hereby, calcium phosphate in high concentration is kept in suspension, resulting in the

\section{KARGER 125}

() 2014 S. Karger AG, Base

0008-6568/14/0491-0056\$39.50/0

E-Mail karger@karger.com

www.karger.com/cre
Dr. Richard J. Wierichs

Department of Operative Dentistry, Periodontology and

Preventive Dentistry (ZPP), RWTH Aachen University, Pauwelsstrasse 30

DE-52074 Aachen (Germany)

E-Mail rwierichs@ukaachen.de 
remineralization of deeper parts of caries lesions also [Reynolds, 1997; Cochrane et al., 2010]. Several in situ studies and clinical trials indicated improved remineralizing effects by CPP-ACP-containing chewing gums (Recaldent ${ }^{\circledR}$ or Topacal ${ }^{\circledR}$ ) [Shen et al., 2001; Iijima et al., 2004; Cai et al., 2007; Morgan et al., 2008], dentifrices [Reynolds et al., 2008; Rao et al., 2009] or creams [Andersson et al., 2007; Bailey et al., 2009; Altenburger et al., 2010] compared with either a negative (without fluoride) or a positive (with fluoride) control. Only a few studies incorporated both control groups [Reynolds et al., 2008; Rao et al., 2009] or included CPP-ACP in varying doses [Shen et al., 2001]. However, in situ studies on chewing gums containing CPP-ACP of other manufacturers could not confirm these positive effects [Itthagarun et al., 2005; Schirrmeister et al., 2007], indicating no beneficial effects of CPP-ACP but resulting in extra costs.

Although reporting a shift of the balance between deand remineralization towards the latter compared with the use of fluoride toothpaste alone, several in vivo studies revealed no distinct positive clinically visible effect for the additional use of CPP-ACP-containing creams. No significant differences in transitions of ICDAS scores were observed between positive control and the additional use of MI Paste (topical remineralizing cream containing CPP-ACP, $10 \% \mathrm{w} / \mathrm{v}$ ) after a period of 12 weeks [Bailey et al., 2009]. Another study [Bröchner et al., 2011] showed no significant difference for visual caries scores and quantitative light-induced fluorescence analysis when the additional use of CPP-ACFP-containing cream (CPP-ACP with fluoride) was compared with fluoride toothpaste alone.

For occlusal surfaces another CPP-ACP-containing cream (Topacal ${ }^{\circledR}$ ) revealed enhanced remineralization compared with brushing with fluoride toothpaste alone over a period of 3 weeks [Altenburger et al., 2010]. This cream promoted regression of post-orthodontic lesions, although only visual evaluation suggested an aesthetically more favourable outcome of the amorphous calcium phosphate treatment for 3 months followed by normal use of fluoride dentifrice for the next 3 months compared with brushing with fluoride dentifrice and use of mouthwash for the entire 6-month period [Andersson et al., 2007].

According to the manufacturer's recommendations GC Tooth Mousse (GC Corporation, Tokyo, Japan) should be applied after brushing with fluoride toothpaste and left for approximately $3 \mathrm{~min}$ to enhance enamel remineralization. However, a remineralizing effect could also be observed if a fluoride toothpaste slurry was not rinsed immediately after tooth brushing [Sjögren et al., 1995]. The effects of CPP-ACP cream on various tooth sites, 'easily cleanable' or 'proximal' (covered with plaque) positions, compared with a prolonged application of fluoride toothpaste have not been studied in an in situ model or in vivo so far. Thus, the aim of this study was to evaluate the remineralizing potential of a fluoride-free CPPACP-containing cream in addition to the use of fluoride toothpaste compared with simple prolonged application of this toothpaste on either brushed or non-brushed enamel caries lesions in situ. We hypothesized that no significant differences in mineral loss would be observed between fluoride-free CPP-ACP-containing cream in addition to the use of fluoride toothpaste and the simple prolonged application of this toothpaste, but that there would be differences for both compared with a non-fluoride (negative) control.

\section{Materials and Methods}

The study design was a double-blind, randomized, cross-over, in situ trial with three treatment legs. Ethical approval was given by the local institutional review board (Christian-Albrechts-Universität zu Kiel; No. A125/09). The number of participants was calculated on the basis of previously performed in situ studies. The dropout rate was assumed not to exceed $10 \%$; completing the study with 11 subjects should have provided at least $99 \%$ power to detect a difference of 957 volume percent $($ vol $\%) \times$ micrometres in mineral loss between treatments on the 'easily cleanable' specimens in a two-sided testing with a 0.05 level of significance. This calculation assumed the root mean square error in the model was $610 \mathrm{vol} \% \times$ micrometres or smaller for mineral loss. All participants gave their written informed consent. They were all in good general health with no signs of active caries or periodontal disease. Exclusion criteria were the following: pregnancy, current participation in another study, institutionalized patients, periodontal disease, active caries lesions, age $<18$ years, salivary flow rate $<0.7 \mathrm{ml} / \mathrm{min}$, no written informed consent, and incapability of contracting. After screening for general eligibility, dental impressions of the lower jaw were taken and appliances were prepared [Koulourides et al., 1974] (fig. 1). The acrylic resin (Orthocryl; Dentaurum, Pforzheim, Germany) of one side of the vestibular flanges was white, indicating that only this flange had to be brushed (see below). In each of both flanges 4 pre-demineralized bovine enamel specimens were inserted [Meyer-Lueckel et al., 2007; Schirrmeister et al., 2007]; 2 'easily cleanable' specimens were fixed with sticky wax and were positioned flush with the acrylic surfaces, and the other 2 specimens were placed in order to form a proximal area and fixed with composite (Tetric EvoFlow; Ivoclar Vivadent, Schaan, Lichtenstein), similar to a previous study [Thomas et al., 2007].

Study Design

The factors under evaluation were as follows:

- Intervention at three levels: (1) application of CPP-ACP-containing cream (non-fluoride, pH 7.8, GC Tooth Mousse,) after 


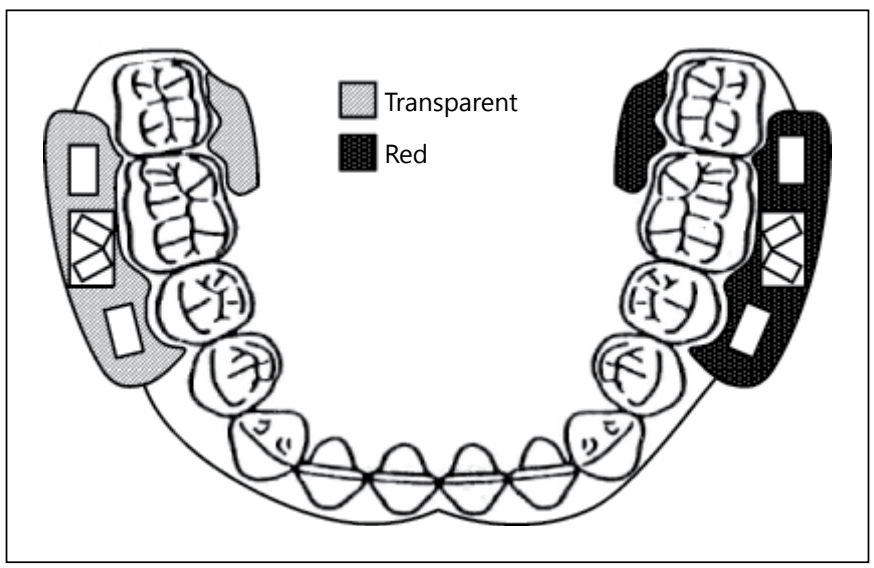

Fig. 1. Design of the intra-oral mandibular appliances. The acrylic resin of 1 side of the vestibular flanges appeared white, indicating that only this flange had to be brushed.

the use of sodium fluoride-containing toothpaste $(1,400 \mathrm{ppm}$ NaF, pH 7.0-7.5, Lavera Zahncreme mint; TM group), (2) prolonged application of fluoride toothpaste (Lavera Zahncreme mint + application of this dentifrice as a cream; positive control, PC group) or (3) prolonged application of fluoride-free toothpaste (non-fluoride, pH 7.0-7.5, Lavera Basis Sensitiv + application of this dentifrice as a cream; negative control, NC group);

- Brushing at two levels: yes (B) or no (NB),

- Position of the specimen at two levels: 'easily cleanable' or 'proximal'

- Localization of the specimen either mesially or distally in the appliance.

The design with an overall of 24 specimens for each participant ensured that a duplet of specimens (for each position) for each of the twelve possible combinations of the three other factors was available for analysis.

GC Tooth Mousse, also known as MI Paste in most nonEuropean countries, is sugar-free and contains $10 \%(\mathrm{w} / \mathrm{v})$ Recal$\operatorname{dent}^{\mathrm{TM}}$ (CPP-ACP). For the PC and NC groups toothpastes of the brand Lavera Basis Sensitiv (Laverana, Wenningsen, Germany) were chosen because of their similar appearance, taste and influence on salivary flow rate as evaluated in a pre-study.

A total of 13 volunteers wore intra-oral mandibular appliances (fig. 1) for three legs each of 4 weeks, the in situ exposure only being interrupted during meals and for oral hygiene. Each of the three treatment legs were immediately preceded by a 1-week 'leanin' period. For each test cycle the participant received a new toothbrush (Oral-B Indicator; Procter \& Gamble, Schwalbach am Taunus, Germany), a nutrition protocol and the dentifrice and cream in neutral packaging. Twice daily during tooth brushing the participants applied either a slurry of fluoride toothpaste (TM and PC groups) or fluoride-free toothpaste (NC group) extra-orally on the specimens for 2 min either with brushing (B) or without brushing (NB). On the vestibular flange to be brushed (transparent resin) the 2 specimens in 'proximal' position were brushed using a proximal brush (diameter: $0.4 \mathrm{~mm}$; TePe, Hamburg, Germany) with 10 movements. The 'easily cleanable' specimens were brushed for $10 \mathrm{~s}$ with the toothbrush. Those of the other flange (red resin) were not brushed at any time to allow plaque to grow. After rinsing, the participants applied GC Tooth Mouse (TM group), fluoride toothpaste as a cream (PC group) or fluoride-free toothpaste as a cream (NC group), respectively, on the specimens, thus simulating the retention of toothpaste without water rinsing immediately after brushing in the PC and NC groups. Moreover, this protocol ensured blinding of the participants. Subsequently, the appliances were re-inserted into the oral cavity and left for at least $30 \mathrm{~min}$ without any intake of drink or food during this period. During additional extra-oral times the appliances were stored in plastic boxes in a humid environment. These extra times were noted and added at the end of each phase to ensure similar wearing times during each phase for each volunteer. The participants were asked not to use fluoride-containing products and received fluoride-free salt for home use. To simulate cariogenic meals the appliances were placed in $10 \%$ sugar solution for 20 min 3 times daily.

\section{Specimen Preparation}

Bovine incisors were obtained from freshly slaughtered cattle (negative BSE test) and stored in $0.08 \%$ thymol. The teeth were cleaned and 746 enamel blocks $(5 \times 3.5 \times 3 \mathrm{~mm})$ were prepared (Exakt 300; Exakt Apparatebau, Norderstedt, Germany). After sterilization with ethylene dioxide, the enamel blocks were embedded in epoxy resin (Technovit 4071; Heraeus Kulzer, Hanau, Germany), ground flat and hand polished (4,000 grit; silicon carbide, Phoenix Alpha; Wirtz-Buehler, Düsseldorf, Germany). The specimens were partially covered with nail varnish (sound control) and subsequently demineralized ( $\mathrm{pH} \mathrm{4.95;} 7$ days) [Buskes et al., 1985]. Thin $(100 \mu \mathrm{m})$ plano-parallel sections were prepared perpendicular to the surface (Trennschleifsystem Exakt 300; Exakt Apparatebau, Norderstedt, Germany) and polished (4,000 grit; Mikroschleifsystem Exakt) to determine the mineral loss at baseline $\left(\Delta \mathrm{Z}_{\text {Baseline }}\right)$. For the in situ period, 312 enamel blocks with similar mineral loss were chosen (see Results below) and inserted into the appliances, mimicking either 'easily cleanable' or 'proximal' surfaces (fig. 1). After the in situ period, thin plano-parallel sections of the same specimens were prepared again, obtaining mineral loss after treatment $\left(\Delta \mathrm{Z}_{\mathrm{Effect}}\right)$.

\section{Transversal Microradiography}

Changes in mineral loss $\left(\Delta \Delta \mathrm{Z}=\Delta \mathrm{Z}_{\text {Baseline }}-\Delta \mathrm{Z}_{\text {Effect }}\right)$ were calculated from transversal microradiographic (TMR) images. Microradiographs of the enamel specimens were obtained using a nickel-copper X-ray source (PW 1730; Philips, Kassel, Germany) operating at $20 \mathrm{kV}$ and $20 \mathrm{~mA}$. The radiation source-to-film distance was $280 \mathrm{~mm}$. The exposure time was $10 \mathrm{~s}$. The film (Fuji 35; Fujifilm, Deutschland) was developed under standardized conditions according to the manufacturer's recommendations. The microradiographs were studied with a digital-image analysing system (CCD Video Camera Modul XC77E; Sony, Tokyo, Japan) that was interfaced with a universal microscope (Axioplan; Zeiss, Oberkochen, Germany) and a personal computer. The contact microradiographs were analysed with respect to mineral losses as well as lesion depths (TMR/WIM Calculation Program, v5.25; University of Groningen, The Netherlands). To minimize the influence of surface blur, the mineral loss and lesion depth values of the sound controls were subtracted from the lesion area data. Changes in integrated mineral loss $\left(\Delta \Delta \mathrm{Z}=\Delta \mathrm{Z}_{\text {Baseline }}-\Delta \mathrm{Z}_{\mathrm{Effect}}\right)$ were calculated. 
Fig. 2. Boxplots with medians and quartiles as well as minima and maxima (circles = outliners) of the changes in mineral losses $(\Delta \Delta \mathrm{Z})$ for various positions - easily cleanable (a), proximal (b). Differences between various subgroups were calculated between those groups differing in either of the independent variables (intervention or brushing procedure). Values were only included when at least 1 specimen of a duplet of a participant could be analysed, therefore $\mathrm{n}=12$. $^{*} \mathrm{p}<0.05$ : Wilcoxon test.

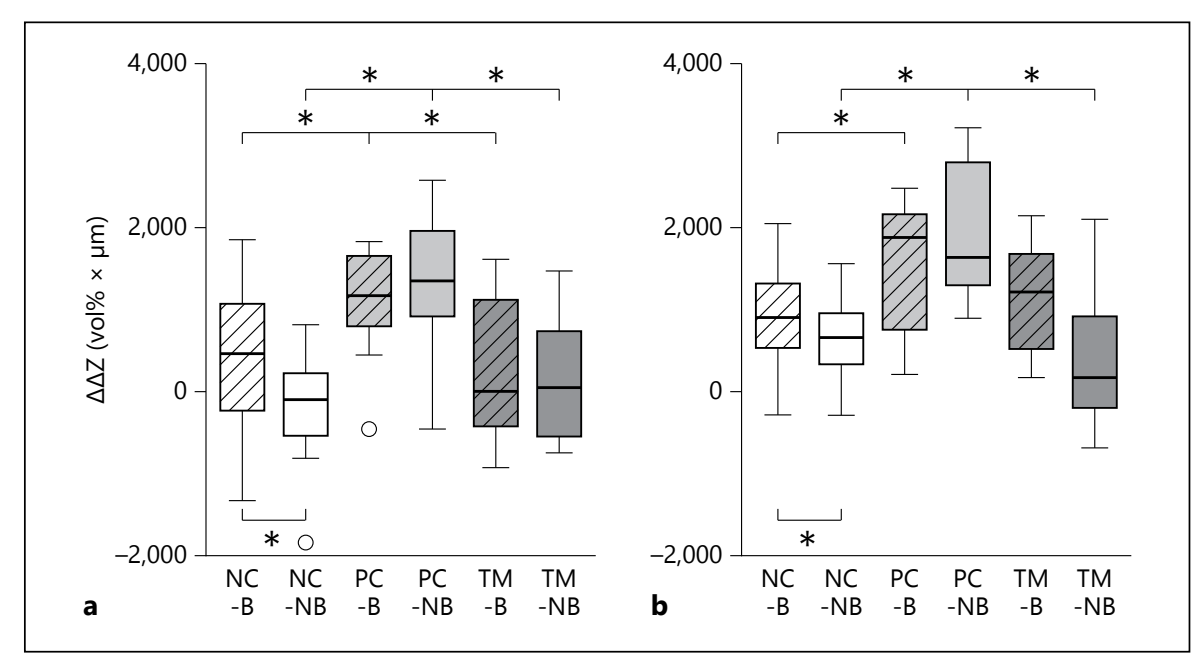

Statistical Analysis

Data were analysed using SPSS statistical software (SPSS 20.0; SPSS, Munich, Germany). Variables were tested for normal distribution (Shapiro-Wilk test). Changes in mineral loss $(\Delta \Delta \mathrm{Z})$ between mesially and distally located samples within the appliance were compared (Wilcoxon test). Due to no significant differences, further analyses were performed with mean values of both localizations. Changes in mineral loss were analysed for those pairs of subgroups differing in only one of the three factors (intervention, brushing and position; Wilcoxon test). Within each subgroup, $\Delta \mathrm{Z}$ values before and after the in situ periods were compared (Wilcoxon test). All tests were performed at a $5 \%$ level of significance.

\section{Results}

All 13 participants ( 4 women and 9 men, aged $22-55$ years) were in good general health. The stimulated salivary flow rate was between 0.7 and $2.1 \mathrm{ml} / \mathrm{min}$. All participants lived in Kiel and used tap water with a fluoride concentration of approximately $0.2 \mathrm{mg} / \mathrm{l}$. The nutrition protocol indicated no consumption of highly fluoridated foods or drinks. No significant differences regarding the consumption of toothpaste and cream, respectively, were observed (PC: 40.2 and $15.2 \mathrm{~g}$; NC: 39.0 and $17.9 \mathrm{~g}$; TM: 32.7 and $12.1 \mathrm{~g} ; \mathrm{p}>0.05$; t-test). However, values were only included when at least 1 specimen of a pair of specimens differing only with respect to the factor position of a participant could be analysed, and therefore 1 participant dropped out.

After pre-demineralization, the samples within the subgroup of the respective position did not differ significantly in mineral loss ( $\mathrm{p}<0.05$; Wilcoxon test). Median (25th/75th percentile) baseline mineral loss was 5,173 $(4,247 / 5,743)$ vol $\% \times$ micrometres and $4,519(4,053 / 5,090)$ vol $\% \times$ micrometres for specimens in the 'easily cleanable' and the 'proximal' positions, respectively. No significant differences in $\Delta \Delta Z$ between 'proximal' and 'easily cleanable' positions except for a borderline significance of the brushed specimen of the TM group ( $\mathrm{p}=0.034$; Wilcoxon test) were observed after in situ exposure. In general, slightly higher remineralization was observed for the 'proximal' position compared with the 'easily cleanable' position.

\section{'Easily Cleanable' Position}

The highest $\Delta \Delta \mathrm{Z}$ value was observed in the PC-NB group (fig. 2). The TM and NC groups showed significantly less remineralization compared with the PC group in brushed and non-brushed specimens ( $p<0.05$; Wilcoxon test). Comparing $\Delta \mathrm{Z}_{\text {Baseline }}$ and $\Delta \mathrm{Z}_{\mathrm{Effect}}$ of a respective group, no significant mineral change could be observed for subgroups of the TM and NC groups ( $p>0.05$; table 1). The brushed specimens of the TM and NC groups revealed a non-significant reduction in mineral loss (remineralization), whereas the non-brushed specimen of those two groups showed a slight but non-significant demineralization. PC-B and PC-NB showed a significant remineralization $(\mathrm{p}<0.05)$.

\section{'Proximal' Position}

The highest remineralization was observed for PC-B (fig. 2). The TM and NC groups showed significantly less remineralization compared with the PC group in brushed and non-brushed specimens, except for the brushed specimens of TM ( $p<0.05$; Wilcoxon test). No significant differences in $\Delta \Delta Z$ between the TM and NC groups for both brushed and non-brushed specimens could be observed 
Table 1. Mineral loss for 'easily cleanable' and 'proximal' positions

\begin{tabular}{|c|c|c|c|c|c|c|}
\hline Position & $\mathrm{n}$ & \multicolumn{2}{|c|}{$\Delta \mathrm{Z}_{\text {Baseline }}(\operatorname{vol} \% \times \mu \mathrm{m})$} & \multicolumn{2}{|c|}{$\Delta \mathrm{Z}_{\text {Effect }}(\operatorname{vol} \% \times \mu \mathrm{m})$} & $\mathrm{p}$ \\
\hline \multicolumn{7}{|c|}{ 'Easily cleanable' } \\
\hline TM-B & 13 & 4,645 & $(4,379 / 5,299)$ & 4,501 & $(3,854 / 4,809)$ & 0.249 \\
\hline TM-NB & 13 & 4,923 & $(4,310 / 5,545)$ & 4,987 & $(3,616 / 5,401)$ & 0.422 \\
\hline PC-B & 12 & 4,914 & $(4,658 / 5,258)$ & 3,598 & $(3,274 / 4,129)$ & 0.003 \\
\hline PC-NB & 13 & 5,321 & $(5,016 / 5,802)$ & 4,276 & $(3,048 / 4,874)$ & 0.002 \\
\hline NC-B & 13 & 5,008 & $(4,379 / 5,299)$ & 4,698 & $(4,211 / 5,060)$ & 0.116 \\
\hline NC-NB & 13 & 5,011 & $(4,310 / 5,545)$ & 5,426 & $(4,484 / 5,952)$ & 0.422 \\
\hline \multicolumn{7}{|l|}{ ‘Proximal’ } \\
\hline TM-B & 12 & 4,549 & $(4,028 / 5,139)$ & 3,594 & $(3,262 / 3,838)$ & 0.002 \\
\hline TM-NB & 13 & 4,666 & $(4,139 / 5,136)$ & 4,069 & $(3,781 / 4,741)$ & 0.133 \\
\hline PC-B & 13 & 4,546 & $(4,088 / 4,979)$ & 2,851 & $(2,253 / 3,697)$ & 0.001 \\
\hline PC-NB & 13 & 4,763 & $(4,356 / 5,211)$ & 3,059 & $(2,411 / 3,549)$ & 0.001 \\
\hline NC-B & 13 & 4,522 & $(4,074 / 4,838)$ & 3,822 & $(3,103 / 3,911)$ & 0.002 \\
\hline NC-NB & 13 & 4,466 & $(4,155 / 4,953)$ & 4,086 & $(3,289 / 4,697)$ & 0.046 \\
\hline
\end{tabular}

Median (25th/75th percentile) mineral loss of specimens in 'easily cleanable' and 'proximal' positions for both brushing procedures: brushed (B) and non-brushed (NB). Italic p-values indicate significant differences in mineral loss before and after the in situ period (Wilcoxon test).

$(\mathrm{p}>0.05)$. Mineral loss after in situ exposure decreased significantly in all subgroups except for specimens of TM-NB ( $<<0.05$; table 1$)$.

\section{Discussion}

The present in situ study aimed to evaluate the remineralizing effect of CPP-ACP-containing cream on mineral loss compared with the effect of the cheaper prolonged retention of fluoride toothpastes. The null hypothesis that no significant differences in mineral loss can be observed between the prolonged use of fluoride toothpaste and the additional use of non-fluoride GC Tooth Mousse could not be confirmed either for the 'easily cleanable' or for 'proximal' positions. In contrast, the additional use of CPP-ACP seemed to even hamper the remineralization that was expected by the use of the fluoride toothpaste.

Brushing the specimens only influenced mineral uptake noticeably in the NC group. This observation confirms previous studies indicating that brushing is beneficial in inhibiting lesion formation and progression in the absence of fluorides [Meyer-Lueckel et al., 2007]. The ability of remineralization by accumulated fluorides in established plaque [Zero et al., 1992] becomes obvious from the comparison of the results of brushed and nonbrushed PC specimens of both positions. Although no brushing was performed, similar remineralization could be observed in the non-brushed compared with the brushed specimens. An elevated fluoride concentration in plaque lasts $12 \mathrm{~h}$ after exposition, whereas the concentration in saliva is reduced to baseline values more quickly, so that low cariogenic plaque can be seen as a deposit of fluoride [Zero et al., 1992].

Brushing with fluoride toothpaste plus the application of CPP-ACP-containing cream induced significantly less remineralization compared with the $\mathrm{PC}$ group. The calcium in the CPP-ACP may have bound the fluorides $\left(\mathrm{CaF}_{2}\right)$ just being incorporated in the enamel surfaces after brushing with the fluoride toothpaste [Cross et al., 2005]. Consequently, $\mathrm{CaF}_{2}$ was dissolved in saliva and swallowed, not being bioavailable during the following demineralization period.

For the TM treatment, a more pronounced mineral gain - being significant for brushed and non-significant for non-brushed specimens - was observed in 'proximal' specimens compared with 'easily cleanable' ones. Calcium ions of the CPP-ACP-containing cream having no fluoride added could possibly bind relatively easily to fluorides in the outer enamel of specimens in the 'easily cleanable' position. Contrastingly, in the 'proximal' position CPP-ACP-containing cream was applied more indirectly onto the specimens, presumably on partially plaque-covered surfaces, since brushing with an interdental brush might not have resulted in complete cleanliness [Slot et al., 2008; Bock et al., 2010], leaving some 
fluoride-containing plaque [Pessan et al., 2006]. This resulted in slightly higher fluoride availability in the case of subsequent demineralization for the 'proximal' specimens compared with the 'easily cleanable' TM specimens. For the NC group, brushing might also not have resulted in complete cleanliness for 'proximal' specimens. However, fluoride concentration in plaque is presumed to be significantly lower after the use of fluoride-free toothpaste than after the use of fluoride toothpaste [Pessan et al., 2006], which results in worse inhibition of demineralization for a fluoride-free control compared with a fluoride toothpaste, as has been observed here. The described effect of fluoride-containing residual plaque is thus not observable in the NC group, as was postulated for TM. Nonetheless, plaque covering enamel lesions might also hamper the diffusion of saliva to the enamel surface, leading to relatively low remineralization [Mellberg, 1992] as observed recently [Cochrane et al., 2012]. Here, the application of CPP-ACP-containing gum was performed for 20 min 5 times a day separately from brushing with fluoride toothpaste over a period of 2 weeks. With this regime for relatively clean surfaces, fluorides were probably incorporated and stabilized in the outer enamel before CPP-ACP could interact with these fluoride ions. Since fluoride incorporation on plaque-retaining surfaces might have been delayed compared with cleaned surfaces, the calcium ions of CPP-ACP could have bound fluorides more easily and for a longer time. Thus, fluorides might not have been bioavailable during the subsequent demineralization periods as well as for remineralization.

The number of subjects who can be included in in situ studies is generally limited [Zero, 1995]. This study population, consisting of 13 participants, seems to be small compared with clinical studies, whereas other in situ studies, indicating improved remineralizing effects by CPP-ACP-containing chewing gums [Iijima et al., 2004; Cai et al., 2007; Cochrane et al., 2012], required only up to 10 participants. Only some studies, querying these positive effects, had a similar number of participants [Itthagarun et al., 2005; Schirrmeister et al., 2007] or many more participants [Shen et al., 2001]. Therefore, the validity of this study compared with other in situ studies is acceptable.

Demineralization depends on the location in the mouth and on the way plaque stagnation is created [Dawes and MacPherson, 1993; Thomas et al., 2007]. In contrast with other in situ studies, in which specimens were situated about $1 \mathrm{~mm}$ recessed to the acrylic and covered with a plastic mesh or gauze [Hara et al., 2003; Itthagarun et al., 2005;
Meyer-Lueckel et al., 2007; Cochrane et al., 2012], in the present study specimens for the 'proximal' position were placed mimicking a proximal space, as performed previously [Thomas et al., 2007]. Thus, plaque retention was supposed to be increased compared with proximal designs of other in situ studies, enhancing lesion progression rates and changes in mineral loss. In the former in situ study plaque formation was promoted by instructing the participants not to clean their samples, which were incorporated in total prosthesis. Nevertheless, in the present study significantly different changes in mineral loss between 'easily cleanable' and 'proximal' specimens could not be observed either for brushed or for non-brushed specimens. Thus, it seems that the chosen design of proximal spaces in the mandibular appliances of volunteers does not lead to an enhanced demineralization as expected.

Former in situ studies demonstrated remineralization by the regular use of CPP-ACP-containing chewing gums for 14 days [Shen et al., 2001]. With this relatively short period natural remineralization from saliva was not exhausted [Neuhaus and Lussi, 2009] and plaque was not allowed to grow at any time. It seems that natural remineralization by saliva only can be enhanced with a study design which lasts at least 21 days [Leach et al., 1989]. Thus, for comparison of CPP-ACP-containing products with those free of CPP-ACP an in situ study length of more than 21 days might show more clinically relevant results.

It can be concluded that only the PC treatment enhanced remineralization significantly within both of the potentially influencing factors (brushing and position) under investigation. CPP-ACP-containing cream with no fluoride showed little beneficial effect in enhancing remineralization. Particularly for plaque-retaining surfaces, CPP-ACP seems to hamper remineralization induced by the use of fluoride toothpaste. CPP-ACFP (with fluoride) might show more positive results with respect to plaque-retaining tooth surfaces, but this has not been studied in situ so far.

\section{Acknowledgement}

We would like to thank the volunteers for participating in the study. The results of this paper have been published in form of the doctoral thesis of T. Schellwien at Christian-Albrechts University of Kiel.

\section{Disclosure Statement}

The authors declare no potential conflicts of interests with respect to the authorship and/or publication of this article. 


\section{References}

Altenburger MJ, Gmeiner B, Hellwig E, Wrbas Dawes C, MacPherson LM: The distribution of KT, Schirrmeister JF: The evaluation of fluorescence changes after application of casein phosphopeptides (CPP) and amorphous calcium phosphate $(\mathrm{ACP})$ on early carious lesions. Am J Dent 2010;23:188-192.

-Andersson A, Skold-Larsson K, Hallgren A, Petersson LG, Twetman S: Effect of a dental cream containing amorphous cream phosphate complexes on white spot lesion regression assessed by laser fluorescence. Oral Health Prev Dent 2007;5:229-233.

Bailey DL, Adams GG, Tsao CE, Hyslop A, Escobar K, Manton DJ, Reynolds EC, Morgan MV: Regression of post-orthodontic lesions by a remineralizing cream. J Dent Res 2009;88: 1148-1153.

Bock NC, von Bremen J, Kraft M, Ruf S: Plaque control effectiveness and handling of interdental brushes during multibracket treatment - a randomized clinical trial. Eur J Orthod 2010;32:408-413.

Bröchner A, Christensen C, Kristensen B, Tranæus S, Karlsson L, Sonnesen L, Twetman S: Treatment of post-orthodontic white spot lesions with casein phosphopeptide-stabilised amorphous calcium phosphate. Clin Oral Investig 2011;15:369-373.

Buskes JA, Christoffersen J, Arends J: Lesion formation and lesion remineralization in enamel under constant composition conditions. A new technique with applications. Caries Res 1985;19:490-496.

Cai F, Manton DJ, Shen P, Walker GD, Cross KJ, Yuan Y, Reynolds C, Reynolds EC: Effect of addition of citric acid and casein phosphopeptide-amorphous calcium phosphate to a sugar-free chewing gum on enamel remineralization in situ. Caries Res 2007;41:377-383.

- Cochrane NJ, Cai F, Huq NL, Burrow MF, Reynolds EC: New approaches to enhanced remineralization of tooth enamel. J Dent Res 2010; 89:1187-1197.

Cochrane NJ, Shen P, Byrne SJ, Walker GD, Adams GG, Yuan Y, Reynolds C, Hoffmann B, Dashper SG, Reynolds EC: Remineralisation by chewing sugar-free gums in a randomised, controlled in situ trial including dietary intake and gauze to promote plaque formation. Caries Res 2012;46:147-155.

Cross KJ, Huq NL, Palamara JE, Perich JW, Reynolds EC: Physicochemical characterization of casein phosphopeptide-amorphous calcium phosphate nanocomplexes. J Biol Chem 2005; 280:15362-15369. saliva and sucrose around the mouth during the use of chewing gum and the implications for the site-specificity of caries and calculus deposition. J Dent Res 1993;72:852-857.

Hara AT, Queiroz CS, Paes Leme AF, Serra MC, Cury JA: Caries progression and inhibition in human and bovine root dentine in situ. Caries Res 2003;37:339-344.

- Iijima Y, Cai F, Shen P, Walker G, Reynolds C, Reynolds EC: Acid resistance of enamel subsurface lesions remineralized by a sugar-free chewing gum containing casein phosphopeptide-amorphous calcium phosphate. Caries Res 2004;38:551-556.

Itthagarun A, King NM, Yiu C, Dawes C: The effect of chewing gums containing calcium phosphates on the remineralization of artificial caries-like lesions in situ. Caries Res 2005; 39:251-254.

Koulourides T, Phantumvanit P, Munksgaard EC, Housch T: An intraoral model used for studies of fluoride incorporation in enamel. J Oral Pathol 1974;3:185-196.

Leach SA, Lee GT, Edgar WM: Remineralization of artificial caries-like lesions in human enamel in situ by chewing sorbitol gum. J Dent Res 1989;68:1064-1068.

Mellberg JR: Hard-tissue substrates for evaluation of cariogenic and anti-cariogenic activity in situ. J Dent Res 1992;71:913-919.

Meyer-Lueckel H, Bitter K, Kielbassa AM: Effect of a fluoridated food item on enamel in situ. Caries Res 2007;41:350-357.

Morgan MV, Adams GG, Bailey DL, Tsao CE, Fischman SL, Reynolds EC: The anticariogenic effect of sugar-free gum containing CPP-ACP nanocomplexes on approximal caries determined using digital bitewing radiography. Caries Res 2008;42:171-184.

Neuhaus KW, Lussi A: Casein phosphopeptideamorphous calcium phosphate (CPP-ACP) and its effect on dental hard tissues. Schweiz Monatsschr Zahnmed 2009;119:110-116.

Paris S, Ekstrand K, Meyer-Lückel H: From diag- Zero DT: In situ caries models. Adv Dent Res nosis to therapy; in Meyer-Lückel H, Paris S, Ekstrand K (eds): Caries Management: Science and Clinical Practice. Stuttgart, Thieme, 2012, pp 133-145.
Pessan JP, Sicca CM, de Souza TS, da Silva SM, Whitford GM, Buzalaf MA: Fluoride concentrations in dental plaque and saliva after the use of a fluoride dentifrice preceded by a calcium lactate rinse. Eur J Oral Sci 2006;114: 489-493.

Rao SK, Bhat GS, Aradhya S, Devi A, Bhat M: Study of the efficacy of toothpaste containing casein phosphopeptide in the prevention of dental caries: a randomized controlled trial in 12- to 15-year-old high caries risk children in Bangalore, India. Caries Res 2009;43:430435.

Reynolds EC: Remineralization of enamel subsurface lesions by casein phosphopeptide-stabilized calcium phosphate solutions. J Dent Res 1997;76:1587-1595.

Reynolds EC, Cai F, Cochrane NJ, Shen P, Walker GD, Morgan MV, Reynolds C: Fluoride and casein phosphopeptide-amorphous calcium phosphate. J Dent Res 2008;87:344-348.

Schirrmeister JF, Seger RK, Altenburger MJ, Lussi A, Hellwig E: Effects of various forms of calcium added to chewing gum on initial enamel carious lesions in situ. Caries Res 2007;41: 108-114.

Shen P, Cai F, Nowicki A, Vincent J, Reynolds EC: Remineralization of enamel subsurface lesions by sugar-free chewing gum containing casein phosphopeptide-amorphous calcium phosphate. J Dent Res 2001;80:2066-2070.

Sjögren K, Birkhed D, Ruben J, Arends J: Effect of post-brushing water rinsing on caries-like lesions at approximal and buccal sites. Caries Res 1995;29:337-342.

Slot DE, Dorfer CE, Van der Weijden GA: The efficacy of interdental brushes on plaque and parameters of periodontal inflammation: a systematic review. Int J Dent Hyg 2008;6:253264.

Thomas RZ, Ruben JL, ten Bosch JJ, Fidler V, Huysmans MC: Approximal secondary caries lesion progression; a 20 -week in situ study. Caries Res 2007;41:399-405.

1995;9:214-230, discussion 231-214.

Zero DT, Raubertas RF, Fu J, Pedersen AM, Hayes AL, Featherstone JD: Fluoride concentrations in plaque, whole saliva, and ductal saliva after application of home-use topical fluorides (published erratum appears in J Dent Res 1993:72(1):87). J Dent Res 1992;71:17681775. 